\title{
A vaccination strategy based on linearization control techniques for fighting against epidemic diseases propagation
}

\author{
Santiago Alonso-Quesada ${ }^{*}$, Manuel De la Sen ${ }^{1}$, Asier Ibeas $^{2}$ and Raúl Nistal ${ }^{1}$
}

\section{"Correspondence:}

santiago.alonso@ehu.es

${ }^{1}$ Department of Electricity and

Electronics, Faculty of Science and

Technology, University of the

Basque Country, UPV/EHU, Campus

de Leioa, Leioa, Bizkaia 48940, Spain

Full list of author information is

available at the end of the article

\begin{abstract}
This paper presents a vaccination strategy for fighting against the propagation of epidemic diseases. The disease propagation is described by a SIRS (susceptible plus infected plus recovered populations) epidemic model. The model takes into account that the recovered individuals lose the disease immunity after a finite time period. A control technique based on a model linearization approach is used to design the vaccination strategy in order to eradicate the infection from the population. Moreover, the controlled system is guaranteed to be positive and stable under such a vaccination control strategy. A simulation example illustrates the theoretical results relative to the stability and positivity of the controlled system while guaranteeing the eradication of the epidemics.

MSC: $93 \mathrm{C} 10 ; 93 \mathrm{C} 15 ; 93 \mathrm{C} 95 ; 34 \mathrm{D} 20 ; 34 \mathrm{H} 15$
\end{abstract}

Keywords: SIRS epidemic models; vaccination; nonlinear control; stability; positivity

\section{Introduction}

The study of propagation of epidemic diseases within a host population has been broadly dealt with for the last decades [1-3]. In this context, several epidemic models have been proposed for analyzing the behavior of different infectious diseases. Among them, the so-called SIRS epidemic model has been used to describe the propagation of epidemic diseases as, for instance, influenza, tetanus, diphtheria, hepatitis A and so on. Such diseases are characterized, among other features, by the fact that the immunization induced by vaccination is only temporary, i.e., such an immunization is not permanent. This feature motivates the use of a SIRS model to explain the disease propagation within the host population [3, 4]. In such a compartmental model, the host population is divided into three categories, each one relative to an epidemic status. Namely, there is a susceptible $(\mathrm{S})$ population composed of either individuals who have not been previously exposed to the pathogen or recovered $(\mathrm{R})$ individuals who have lost the immunity after having successfully cleared the infection. Finally, there is an infected (I) population composed of individuals who are currently colonized by the pathogen. Moreover, there are three transitions between such population categories. The first one is the transition from the susceptible population to the infected one. Such a transition occurs when there is a contact between a susceptible individual and an infected one. In such situations there is a probability of disease transmission from the infected individual to the susceptible one who becomes infected after a latent period. This latent period is not taken into account in a SIRS model.

\section{Springer}

(O2013 Alonso-Quesada et al: licensee Springer. This is an Open Access article distributed under the terms of the Creative Commons Attribution License (http://creativecommons.org/licenses/by/2.0), which permits unrestricted use, distribution, and reproduction in any medium, provided the original work is properly cited. 
However, the model can be extended to include such a time period giving place to a SEIRS model [2]. The transition from the infected category to the recovered one occurs when an infected individual has fought off the infection. Such a transition occurs after an infection period. Finally, the transition from the recovered category to the susceptible one occurs when a recovered individual loses the temporary immunity [1].

One of the purposes of analyzing epidemic models concerns the design of control strategies in order to eradicate the persistence of the infection in the host population. In this context, constant, regular and/or impulsive vaccination strategies have been proposed in several research papers [5-10]. Concretely, a regular vaccination strategy based on a feedback control law for exact input-output linearization of a SEIR epidemic model is designed in [8]. Moreover, such a control strategy is combined with an observer in [9]. The observer is designed to estimate on-line the susceptible and the infected (or exposed) populations since such measures are not available in a real situation where only the infectious population is measurable. The estimates provided by the observer are used to synthesize the control law instead of the true susceptible and infected populations. Such strategies achieve the eradication of the disease from the host population while guaranteeing the positivity and stability of the controlled system. Other potential situation is that the parameters of the epidemic model are not fully known, which may be circumvented by using adaptive control strategies [11].

Linearization and impulsive control techniques have been satisfactorily used for stabilizing highly nonlinear processes in applications of diverse nature. On the one hand, an active magnetic bearing system composed of a spindle rotating at different speeds is stabilized by means of a control strategy based on a linearization of the process in [12]. On the other hand, a fuzzy impulsive strategy is designed to achieve the stabilization in chaos control in [13]. Also, sliding mode control techniques have been applied in models of diseases propagation [14]. This use is motivated by the satisfactory results obtained in the application of such techniques in other kind of systems [15].

The proposed SEIR model in $[8,9]$ assumes that the whole host population is maintained constant for all time, i.e., such a model is valid when the mortality by causes relative to the disease is negligible, and the birth and naturally mortality rates are similar so that the whole host population may be considered time-invariant. In the same line, the current paper proposes a vaccination strategy based on a linearization control technique of the mapping from the vaccination control to the infected population in order to eradicate an epidemic disease propagation of which can be described by a SIRS epidemic model. The control law based on an input-output linearization technique is useful in order to design a vaccination strategy since the epidemic models in general, and then the SIRS model considered in this paper, are nonlinear systems. Concretely, they include a bilinear term which describes the potential disease transmission when contacts between susceptible and infected individuals happen. The main difference of the current research with respect to $[8,9]$ is that the mortality from causes relative to the infection is appreciable. Also, the birth and mortality from nature causes may be different so that the whole host population may be time-varying. A normalized SIRS epidemic model is used to circumvent the complexity from the fact that the whole population is time-varying. Such a normalized model is obtained from the original SIRS one via a suitable variables change. Moreover, such a normalized model is used to synthesize the vaccination control law which ensures the eradication of the infection from the host population and the positivity of the normalized SIRS 
model as well as the original SIRS epidemic model. The mathematical proofs of the epidemics eradication and the positivity of both models constitute the main contribution of this paper. Also, a simulation example based on a rabbit hemorrhagic disease illustrates the theoretical results. Such a disease has been chosen in such a numerical study because of its high mortality [16].

Finally, the described control strategy may be extended to more complex epidemical models which consider more population categories than the presented SIRS model. In this sense, vaccinated, quarantine, susceptible population with different risk of catching infection, asymptomatic or symptomatic infected populations have been considered in other compartmental models for describing the propagation of infectious diseases within a host population [17-19].

Notation $\mathbb{R}_{+} \triangleq(0, \infty) \cap \mathbb{R}$ is the set of strictly positive real numbers and $\mathbb{R}_{0_{+}} \triangleq \mathbb{R}_{+} \cup\{0\}$. $\mathbb{R}_{-} \triangleq(-\infty, 0) \cap \mathbb{R}$ is the set of strictly negative real numbers and $\mathbb{R}_{0_{-}} \triangleq \mathbb{R}_{-} \cup\{0\}$. $\mathbb{R}_{+}^{2}$ is the first open real quadrant and $\mathbb{R}_{0+}^{2}$ is the first closed real quadrant. $\mathbb{R}_{-}^{2}$ is the third open real quadrant and $\mathbb{R}_{0-}^{2}$ is the third closed real quadrant.

\section{SIRS epidemic model}

Let $S(t), I(t)$ and $R(t)$ be, respectively, the susceptible, infected and recovered or removedby-immunity populations at time $t$. Consider a time-invariant SIRS epidemic model given by

$$
\begin{aligned}
& \dot{S}(t)=-\mu S(t)+\omega R(t)-\beta \frac{S(t) I(t)}{N(t)}+v N(t)[1-V(t)], \\
& \dot{I}(t)=-(\mu+\gamma) I(t)+\beta \frac{S(t) I(t)}{N(t)}, \\
& \dot{R}(t)=-(\mu+\omega) R(t)+\gamma(1-\rho) I(t)+v N(t) V(t)
\end{aligned}
$$

subject to the initial conditions $S(0) \geq 0, I(0) \geq 0$ and $R(0) \geq 0$ under a vaccination function $V: \mathbb{R}_{0_{+}} \rightarrow \mathbb{R}_{0_{+}}$. In the above SIRS model, $N(t) \geq 0$ is the total population at any time instant $t \in \mathbb{R}_{0_{+}}, \mu>0$ is the death rate from natural causes unrelated to the infection, $v>0$ is the birth rate, $\omega>0$ is the rate of losing immunity, $\beta>0$ is the transmission constant (with the total number of infections per unity of time at time $t$ being $\beta S(t) I(t) / N(t)$ ), $\gamma>0$ is the recovery rate (or $\gamma^{-1}$ the average duration of the infective period) and $\rho \in[0,1)$ is the probability of death from infection causes. The total population dynamics is obtained by summing up (1)-(3) yielding

$$
\dot{N}(t)=\dot{S}(t)+\dot{I}(t)+\dot{R}(t)=(\nu-\mu) N(t)-\rho \gamma I(t)
$$

so that the total population is time-varying. A normalized SIRS model is used to analyze and design a vaccination control strategy.

\subsection{Normalized SIRS model}

The SIRS model (1)-(3) is normalized with respect to the whole population by using the following variables change:

$$
s(t)=\frac{S(t)}{N(t)} ; \quad \iota(t)=\frac{I(t)}{N(t)} ; \quad r(t)=\frac{R(t)}{N(t)} .
$$


By introducing this variables change in (1)-(3), one obtains the normalized SIRS model given by

$$
\begin{aligned}
& \dot{s}(t)=-v s(t)+\omega r(t)+(\rho \gamma-\beta) s(t) \iota(t)+v[1-V(t)], \\
& i(t)=-(v+\gamma) \iota(t)+\beta s(t) \iota(t)+\rho \gamma \iota(t)^{2}, \\
& \dot{r}(t)=-(v+\omega) r(t)+\gamma(1-\rho) \iota(t)+\rho \gamma \iota(t) r(t)+v V(t) .
\end{aligned}
$$

By summing up (6)-(8), it follows that $\dot{s}(t)+i(t)+\dot{r}(t)=0$ so that $s(t)+\iota(t)+r(t)=1$ for all time irrespective of the vaccination function.

\section{Vaccination strategy}

The main control objective is that the infected population asymptotically tends to zero as $t \rightarrow \infty$, so the infection is eradicated from the population, while guaranteeing the positivity of the controlled system. A vaccination control law based on a static-state feedback linearization strategy is developed for achieving such a control objective. This technique requires a nonlinear coordinate transformation, based on the Lie derivatives theory, in the system representation [20].

The dynamics equations (6)-(8) of the normalized SIRS model can be equivalently written as the following nonlinear control affine system:

$$
\dot{\mathbf{x}}(t)=\mathbf{f}(\mathbf{x}(t))+\mathbf{g}(\mathbf{x}(t)) u(t) ; \quad y(t)=h(\mathbf{x}(t))
$$

where $y(t)=\iota(t) \in \mathbb{R}_{0_{+}}, u(t)=V(t) \in \mathbb{R}_{0_{+}}$and $\mathbf{x}(t)=[\iota(t) s(t)]^{T} \in \mathbb{R}_{0_{+}}^{2}$ are, respectively, considered as the output signal, the input signal and the state vector of the system $\forall t \in \mathbb{R}_{0_{+}}$, and $r(t)=1-s(t)-\iota(t)$ has been used with

$$
\begin{aligned}
& \mathbf{f}(\mathbf{x}(t))=\left[\begin{array}{c}
-(v+\gamma) \iota(t)+\beta s(t) \iota(t)+\rho \gamma \iota^{2}(t) \\
-(v+\omega) s(t)-\omega \iota(t)+(\rho \gamma-\beta) s(t) \iota(t)+v+\omega
\end{array}\right] \in \mathbb{R}^{2} ; \\
& \mathbf{g}(\mathbf{x}(t))=\left[\begin{array}{cc}
0 & -v
\end{array}\right]^{T} \in \mathbb{R}_{0-}^{2} ; \quad h(\mathbf{x}(t))=\iota(t) \in \mathbb{R}_{0+} .
\end{aligned}
$$

The first step to apply a coordinate transformation based on the Lie derivation is to determine the relative degree of the system. For such a purpose, the following definitions are taken into account: (i) $L_{\mathbf{f}}^{k} h(\mathbf{x}(t)) \triangleq \frac{\partial\left(L_{\mathbf{f}}^{k-1} h(\mathbf{x}(t))\right)}{\partial \mathbf{x}} \mathbf{f}(\mathbf{x}(t))$ is the $k$ th-order Lie derivative of $h(\mathbf{x}(t))$ along $\mathbf{f}(\mathbf{x}(t))$ with $L_{\mathbf{f}}^{0} h(\mathbf{x}(t)) \triangleq h(\mathbf{x}(t))$ and (ii) the relative degree $r$ of the system is the number of times that the output must be differentiated to obtain the input explicitly, i.e., the number $r$ so that $L_{\mathbf{g}} L_{\mathbf{f}}^{k} h(\mathbf{x}(t))=0$ for $k<r-1$ and $L_{\mathbf{g}} L_{\mathbf{f}}^{r-1} h(\mathbf{x}(t)) \neq 0$.

From (10), $L_{\mathbf{g}} h(\mathbf{x}(t))=0$, while $L_{\mathbf{g}} L_{\mathbf{f}} h(\mathbf{x}(t))=-v \beta \iota(t)$, so the relative degree of the system is 2 in $D \triangleq\left\{\mathbf{x}=[\iota s]^{T} \in \mathbb{R}_{0+}^{2} \mid \iota \neq 0\right\}$, i.e., $\forall \mathbf{x} \in \mathbb{R}_{0+}^{2}$ except in the singular surface $\iota=0$ of the state space where the relative degree is not well defined. Since the relative degree of the system is exactly equal to the dimension of the state space for any $\mathbf{x} \in D$, the nonlinear coordinate change

$$
\bar{\iota}(t)=L_{\mathbf{f}}^{0} h(\mathbf{x}(t))=\iota(t) ; \quad \bar{s}(t)=L_{\mathbf{f}} h(\mathbf{x}(t))=-(v+\gamma) \iota(t)+\beta s(t) \iota(t)+\rho \gamma \iota^{2}(t)
$$


allows to represent the model in the so-called normal form in a neighborhood of any $\mathbf{x} \in D$. Namely,

$$
\dot{\overline{\mathbf{x}}}(t)=\overline{\mathbf{f}}(\overline{\mathbf{x}}(t))+\overline{\mathbf{g}}(\overline{\mathbf{x}}(t)) u(t) ; \quad y(t)=h(\overline{\mathbf{x}}(t))
$$

where $\overline{\mathbf{x}}(t)=[\bar{l}(t) \bar{s}(t)]^{T}$ and

$$
\begin{aligned}
\overline{\mathbf{f}}(\overline{\mathbf{x}}(t))= & {[\bar{s}(t) \quad \phi(\overline{\mathbf{x}}(t))]^{T} ; \quad \overline{\mathbf{g}}(\overline{\mathbf{x}}(t))=\left[\begin{array}{ll}
0 & -v \beta \bar{\imath}(t)
\end{array}\right]^{T} ; \quad h(\overline{\mathbf{x}}(t))=\bar{\iota}(t)=\iota(t) ; } \\
\phi(\overline{\mathbf{x}}(t))= & (\beta-v-\gamma)(v+\omega) \bar{\imath}(t)-(v+\omega) \bar{s}(t) \\
& +(\rho \gamma(2 v+\omega+\gamma)-\beta(v+\omega+\gamma)) \bar{\iota}^{2}(t) \\
& +(2 \rho \gamma-\beta) \bar{s}(t) \bar{\iota}(t)+\frac{\bar{s}^{2}(t)}{\bar{\imath}(t)}-\rho \gamma(\rho \gamma-\beta) \bar{\iota}^{3}(t) .
\end{aligned}
$$

The following result being relative to the input-output linearization of the system is established.

Theorem 1 The state feedback control law

$$
u(t)=\frac{-L_{\mathbf{f}}^{2} h(\mathbf{x}(t))-\lambda_{0} h(\mathbf{x}(t))-\lambda_{1} L_{\mathbf{f}} h(\mathbf{x}(t))}{L_{\mathbf{g}} L_{\mathbf{f}} h(\mathbf{x}(t))},
$$

where $\lambda_{0}$ and $\lambda_{1}$ are the controller tuning parameters, induces the linear closed-loop $d y$ namics

$$
\ddot{y}(t)+\lambda_{1} \dot{y}(t)+\lambda_{0} y(t)=0
$$

around any point $\mathbf{x} \in D$.

Proof The state equation for the closed-loop system

$$
\left[\begin{array}{c}
\dot{\bar{l}}(t) \\
\dot{\bar{s}}(t)
\end{array}\right]=\left[\begin{array}{c}
\bar{s}(t) \\
\phi(\overline{\mathbf{x}}(t))-L_{\mathbf{f}}^{2} h(\mathbf{x}(t))-\lambda_{0} \bar{l}(t)-\lambda_{1} \bar{s}(t)
\end{array}\right]
$$

is obtained by introducing the control law (14) in (12) and taking into account the fact that $L_{\mathbf{g}} L_{\mathbf{f}} h(\mathbf{x}(t))=-v \beta \iota(t)=-v \beta \bar{\iota}(t) \neq 0 \forall \mathbf{x} \in D$ and the coordinate transformation (11). Also, it follows that $L_{\mathrm{f}}^{2} h(\mathbf{x}(t))=\phi(\overline{\mathbf{x}}(t))$ by direct calculations. Thus, the state equation of the closed-loop system in the state space defined by $\overline{\mathbf{x}}(t)$ can be written as

$$
\dot{\overline{\mathbf{x}}}(t)=\mathbf{A} \overline{\mathbf{x}}(t) \quad \text { with } \mathbf{A}=\left[\begin{array}{cc}
0 & 1 \\
-\lambda_{0} & -\lambda_{1}
\end{array}\right] .
$$

Furthermore, the output equation of the closed-loop system is $y(t)=\mathbf{C} \overline{\mathbf{x}}(t)$ with $\mathbf{C}=\left[\begin{array}{ll}1 & 0\end{array}\right]$ since $y(t)=\iota(t)=\bar{l}(t)$. From (17) and the closed-loop output equation, it follows that

$$
\begin{aligned}
\ddot{y}(t) & =\ddot{\bar{l}}(t)=\dot{\bar{s}}(t)=-\lambda_{0} \bar{l}(t)-\lambda_{1} \bar{s}(t) \\
& =-\lambda_{0} y(t)-\lambda_{1} \dot{y}(t) \quad \Rightarrow \quad \ddot{y}(t)+\lambda_{1} \dot{y}(t)+\lambda_{0} y(t)=0 .
\end{aligned}
$$


Remark 1 The roots of the characteristic polynomial $P(s)=s^{2}+\lambda_{1} s+\lambda_{0}$ corresponding to the closed-loop dynamics (15) are given by $p_{1,2}=\frac{-\lambda_{1} \pm \sqrt{\lambda_{1}^{2}-4 \lambda_{0}}}{2}$. Then the stability of the closed-loop dynamics is guaranteed if the control parameters are chosen strictly positive so that such roots have real parts being strictly negative. Moreover, such a choice implies the exponential convergence to zero of the output variable $\iota(t)=\bar{l}(t)$ as time tends to infinity and, as a consequence, the eradication of the infection from the host population. However, the control parameters choice also has to guarantee the positivity of the susceptible, infected and recovered populations for all time as the nature of the system requires. This constraint implies that the model variables $\iota(t)$ and $s(t)$ have to be such that $\iota(t)+s(t) \in[0,1] \forall t \in \mathbb{R}_{0+}$ so that $r(t) \in[0,1] \forall t \in \mathbb{R}_{0_{+}}$in view of the constraint $\iota(t)+s(t)+r(t)=1 \forall t \in \mathbb{R}_{0+}$. Such a positivity property implies additional conditions to be satisfied by the controller parameters $\lambda_{0}$ and $\lambda_{1}$. This analysis is carried out in Section 3.1 below.

\subsection{Control parameters choice}

The application of the control law (14), obtained from the exact input-output linearization strategy, makes the closed-loop dynamics of the normalized infected population be given by (15). Such a dynamics depends on the control parameters $\lambda_{0}$ and $\lambda_{1}$. Such parameters have to be appropriately chosen in order to guarantee the following suitable properties: (i) the stability and positivity of the controlled SIRS model, (ii) the eradication of the infection, i.e., the asymptotic convergence of $I(t)$ to zero as time tends to infinity and (iii) the non-negativity of the vaccination function for all time. The following theorems related to the choice of the controller tuning parameters in order to meet such properties are proven.

Theorem 2 Assume that the initial condition $\mathbf{x}(0)=[\iota(0) s(0)]^{T} \in \mathbb{R}_{0_{+}}^{2}$ fulfils $\iota(0) \in[0,1]$, $s(0) \in[0,1]$ and $\iota(0)+s(0) \in[0,1]$. Moreover, both roots $p_{1}$ and $p_{2}$ of the characteristic polynomial $P(s)$ associated with the closed-loop dynamics (15) are of strictly negative real part via an appropriate choice of the free-design controller parameters $\lambda_{0}$ and $\lambda_{1}$. Then the control law (14) guarantees the exponential stability of the transformed controlled SIRS model (12)-(13). Moreover, the normalized SIRS model (6)-(8) has the following properties: $\iota(t), s(t) \iota(t)$ and $s(t)+r(t)$ are bounded for all time, $\iota(t) \rightarrow 0, s(t)+r(t) \rightarrow 1$ and $s(t) \iota(t) \rightarrow 0$ exponentially as $t \rightarrow \infty$, and $\iota(t)=o(1 / s(t))$.

Proof The dynamics of the normalized controlled SIRS model (15) can be equivalently written with the state equation (17) and the output equation $y(t)=\mathbf{C} \overline{\mathbf{x}}(t)$, where $\mathbf{C}=\left[\begin{array}{ll}1 & 0\end{array}\right]$, by taking into account that $y(t)=\bar{l}(t)$ and $\dot{y}(t)=\bar{s}(t)$. The initial condition $\overline{\mathbf{x}}(0)=[\bar{l}(0) \bar{s}(0)]^{T}$ in such a realization is bounded since it is related to $\mathbf{x}(0)$ via the coordinate transformation (11) and $\mathbf{x}(0)$ is bounded. Such a controlled model is exponentially stable since the eigenvalues of the matrix $\mathbf{A}$ are the roots $p_{1}$ and $p_{2}$ of $P(s)$ which are assumed to be in the open left-half complex plane. Then the state vector $\overline{\mathbf{x}}(t)$ exponentially converges to zero as $t \rightarrow \infty$ while being bounded for all time. It implies that $\iota(t)$ is bounded for all time and converges exponentially to zero as $t \rightarrow \infty$ from the boundedness and exponential convergence to zero of $\overline{\mathbf{x}}(t)$ as $t \rightarrow \infty$ since $\iota(t)=\bar{\imath}(t)$. Furthermore, the boundedness of $s(t)+r(t)$ for all time and its exponential convergence to unity as $t \rightarrow \infty$ are derived from the boundedness of $\iota(t)$, the exponential convergence to zero of $\iota(t)$ as $t \rightarrow \infty$ and the fact that $\iota(t)+s(t)+r(t)=1 \forall t \in \mathbb{R}_{0_{+}}$. Finally, from the second equation of (11), it follows that 
$s(t) \iota(t)$ is bounded and it exponentially converges to zero as $t \rightarrow \infty$ from the boundedness and exponential convergence to zero of $\iota(t)$ and $\overline{\mathbf{x}}(t)$ as $t \rightarrow \infty$. The facts that $\iota(t) \rightarrow 0$ and $s(t) \iota(t) \rightarrow 0$ as $t \rightarrow \infty$ imply directly that $\iota(t)=o(1 / s(t))$.

Theorem 3 Assume that the parameters and the initial condition of a SIRS epidemic model describing the propagation of an epidemic disease satisfy that

$$
\begin{aligned}
& N(0)>0 ; \quad \beta>\operatorname{Max}\{v+\gamma, 2 \rho \gamma\} ; \quad 0<\frac{v+\gamma(1-\rho \iota(0))}{\beta}<s(0) \leq 1 ; \\
& 0 \leq \iota(0)<\operatorname{Min}\left\{\frac{\beta-v-\gamma}{\beta-\rho \gamma}, \frac{(\beta-v-\gamma)(v+\omega)}{(\beta-\rho \gamma)(v+\gamma+\omega)-\rho \gamma \nu}\right\}<1 .
\end{aligned}
$$

If a vaccination strategy based on the control law (14) with the tuning parameters $\lambda_{0}$ and $\lambda_{1}$ is chosen such that the poles $p_{1}$ and $p_{2}$ of the controlled normalized system satisfy

$$
\begin{aligned}
& -(v+\gamma(1-\rho \varepsilon \iota(0))) \leq p_{1}<\operatorname{Min}\{0, \mu-v\} \\
& p_{2}=\frac{1}{\varepsilon-1}\left(\varepsilon p_{1}+v+\gamma-\beta s(0)-\rho \gamma \iota(0)\right)<p_{1}
\end{aligned}
$$

for some real parameter $\varepsilon \in\left(1, \varepsilon_{\max }\right)$ where the upper-bound is given by

$$
\varepsilon_{\max }=\operatorname{Min}\left\{\frac{v+\gamma}{\rho \gamma \iota(0)}, \frac{\beta s(0)+\rho \gamma \iota(0)}{p_{1}+v+\gamma}, \bar{\varepsilon}_{1}\left(p_{1}\right), \bar{\varepsilon}_{2}\left(p_{1}\right)\right\}
$$

with

$$
\begin{aligned}
& \bar{\varepsilon}_{1}\left(p_{1}\right)= \begin{cases}\frac{(\beta s(0)+\rho \gamma \iota(0)-\nu-\gamma) p_{1}-g_{1}(\cdot)}{p_{1}^{2}-g_{1}(\cdot)} & \text { if } p_{1}^{2}<g_{1}(\cdot), \\
\infty & \text { otherwise }\end{cases} \\
& \bar{\varepsilon}_{2}\left(p_{1}\right)= \begin{cases}\frac{g_{2}(\cdot)(\beta s(0)+\rho \gamma \iota(0)-\nu-\gamma)}{g_{2}(\cdot) p_{1}+\left(\beta s(0)+\rho \gamma \iota(0)-\nu-\gamma-p_{1}\right) g_{3}(\cdot) \iota(0)} & \text { if } p_{1}>-\frac{(\beta s(0)+\rho \gamma(0)-\nu-\gamma) g_{3}(\cdot) \iota(0)}{g_{2}(\cdot)-g_{3}(\cdot) \iota(0)} \\
\infty & \text { otherwise, }\end{cases}
\end{aligned}
$$

where

$$
\begin{aligned}
& g_{1}(\beta, v, \gamma, \rho)=\beta(\beta-v-2 \rho \gamma)+\rho \gamma(v+\gamma) ; \\
& g_{2}(\beta, v, \gamma, \omega)=(\beta-v-\gamma)(v+\omega) ; \\
& g_{3}(\beta, v, \gamma, \omega, \rho)=(\beta-\rho \gamma)(v+\gamma+\omega)-\rho \gamma v,
\end{aligned}
$$

then:

(i) the normalized populations are non-negative $\forall t \in \mathbb{R}_{0_{+}}$, i.e., $0 \leq \iota(t) \leq 1,0 \leq s(t) \leq 1$ and $0 \leq r(t) \leq 1 \forall t \in \mathbb{R}_{0+}$,

(ii) the populations $I(t), S(t), R(t)$ and $N(t)$ are non-negative $\forall t \in \mathbb{R}_{0_{+}}$,

(iii) the epidemics is eradicated from the population, i.e., $I(t)$ tends asymptotically to zero as $t \rightarrow \infty$, and

(iv) the vaccination control function is non-negative $\forall t \in \mathbb{R}_{0_{+}}$, i.e., $u(t)=V(t) \geq 0$ $\forall t \in \mathbb{R}_{0+}$. 
Proof (i) The dynamics of the normalized controlled SIRS model (15) can be equivalently written with the state equation (17) and the output equation $y(t)=\mathbf{C} \overline{\mathbf{x}}(t)$, where $\mathbf{C}=\left[\begin{array}{ll}1 & 0\end{array}\right]$, by taking into account that $y(t)=\bar{\imath}(t)$ and $\dot{y}(t)=\bar{s}(t)$. From such a realization and taking into account the first equation in (11) it follows that

$$
\iota(t)=\bar{\imath}(t)=y(t)=c_{1} e^{p_{1} t}+c_{2} e^{p_{2} t}
$$

$\forall t \in \mathbb{R}_{0+}$ for some constants $c_{1}$ and $c_{2}$ being dependent on the initial conditions $y(0)=\bar{l}(0)$ and $\dot{y}(0)=\bar{s}(0)$, and where $p_{1}$ and $p_{2}$ denote the eigenvalues of $\mathbf{A}$, i.e., the roots of the characteristic polynomial $P(s)=s^{2}+\lambda_{1} s+\lambda_{0}$, which may be fixed to desired values by appropriately adjusting the control parameters $\lambda_{0}$ and $\lambda_{1}$. The values $\bar{l}(0)$ and $\bar{s}(0)$ are related to the initial conditions of the normalized SIRS model in its original realization, i.e., in the state space defined by $\mathbf{x}(t)=[\iota(t) s(t)]^{T}$ via (11). In this way, the constants $c_{1}$ and $c_{2}$ can be obtained by solving the following set of linear equations:

$$
\begin{aligned}
& y(0)=\bar{\iota}(0)=c_{1}+c_{2}=\iota(0) ; \\
& \dot{y}(0)=\bar{s}(0)=c_{1} p_{1}+c_{2} p_{2}=-(v+\gamma) \iota(0)+\beta s(0) \iota(0)+\rho \gamma \iota^{2}(0),
\end{aligned}
$$

where (11) and (23) have been used. One obtains directly from (24) and by taking into account the assignment of $p_{2}$ in (20) that

$$
\begin{aligned}
& c_{1}=\frac{\beta s(0) \iota(0)+\rho \gamma \iota^{2}(0)-\left(p_{2}+v+\gamma\right) \iota(0)}{p_{1}-p_{2}}=\varepsilon \iota(0) ; \\
& c_{2}=\iota(0)-c_{1}=(1-\varepsilon) \iota(0) .
\end{aligned}
$$

Then it follows from (23) and (25) that

$$
\iota(t)=\varepsilon \iota(0)\left(e^{p_{1} t}-e^{p_{2} t}\right)+\iota(0) e^{p_{2} t} \geq 0 \quad \forall t \in \mathbb{R}_{0+}
$$

since $e^{p_{1} t}-e^{p_{2} t} \geq 0 \forall t \in \mathbb{R}_{0+}$ from the fact that $p_{2}<p_{1}<0$ subject to the constraints (19)(21). From (11), (23), (25) and the facts that $\bar{s}(t)=\dot{y}(t)$ and $0 \leq \iota(t) \leq \varepsilon \iota(0) e^{p_{1} t}$, which implies that $0 \leq \iota^{2}(t) \leq \varepsilon^{2} \iota^{2}(0) e^{2 p_{1} t} \leq \varepsilon^{2} \iota^{2}(0) e^{p_{1} t}$ since $p_{1}<0$, it follows that

$$
\begin{aligned}
\beta s(t) \iota(t) & =\bar{s}(t)+(v+\gamma) \iota(t)-\rho \gamma \iota^{2}(t) \\
& \geq \varepsilon \iota(0)\left(p_{1}+v+\gamma-\rho \gamma \varepsilon \iota(0)\right) e^{p_{1} t}+(1-\varepsilon) \iota(0)\left(p_{2}+v+\gamma\right) e^{p_{2} t} \geq 0
\end{aligned}
$$

$\forall t \in \mathbb{R}_{0+}$, since $p_{1}+v+\gamma-\rho \gamma \varepsilon l(0) \geq 0$ from $(20), \varepsilon>1$ and

$$
p_{2}+v+\gamma=\frac{\varepsilon\left(p_{1}+v+\gamma\right)-\beta s(0)-\rho \gamma \iota(0)}{\varepsilon-1}<0
$$

by taking into account (20) and the fact that $\varepsilon<\frac{\beta s(0)+\rho \gamma \iota(0)}{p_{1}+v+\gamma}$ from (21). The fact that $\iota(t) \geq 0$ $\forall t \in \mathbb{R}_{0+}$ and (27) implies that $s(t) \geq 0 \forall t \in \mathbb{R}_{0_{+}}$since $\beta>0$. From the fact that $\iota(t)+s(t)+$ $r(t)=1 \forall t \in \mathbb{R}_{0+}$ and (11), it follows that

$$
r(t)=1-\iota(t)-s(t)=\frac{(\beta-v-\gamma) \iota(t)-(\beta-\rho \gamma) \iota^{2}(t)-\bar{s}(t)}{\beta \iota(t)}
$$


$\forall t \in \mathbb{R}_{0_{+}}$, and then

$$
\begin{aligned}
\beta \iota(t) r(t)= & (\beta-v-\gamma) \iota(0)\left(\varepsilon e^{p_{1} t}+(1-\varepsilon) e^{p_{2} t}\right)-(\beta-\rho \gamma) \iota^{2}(0)\left(\varepsilon e^{p_{1} t}+(1-\varepsilon) e^{p_{2} t}\right)^{2} \\
& -\iota(0)\left(p_{1} \varepsilon e^{p_{1} t}+p_{2}(1-\varepsilon) e^{p_{2} t}\right)
\end{aligned}
$$

$\forall t \in \mathbb{R}_{0_{+}}$by taking into account (23), (25) and that $\bar{s}(t)=\frac{d}{d t}(\iota(t))$. By introducing the expression (20) for $p_{2}$ in (30), one obtains that

$$
\beta \iota(t) r(t)=f_{1}(t)-f_{2}(t)-f_{3}(t)
$$

$\forall t \in \mathbb{R}_{0_{+}}$, where

$$
\begin{aligned}
& f_{1}(t)=\varepsilon\left(\beta-v-\gamma-p_{1}\right) \iota(0) e^{p_{1} t} ; \\
& f_{2}(t)=\left((\varepsilon-1) \beta+\beta s(0)+\rho \gamma \iota(0)-\varepsilon\left(\nu+\gamma+p_{1}\right)\right) \iota(0) e^{p_{2} t} ; \\
& f_{3}(t)=(\beta-\rho \gamma) \iota^{2}(0)\left(\varepsilon e^{p_{1} t}+(1-\varepsilon) e^{p_{2} t}\right)^{2} .
\end{aligned}
$$

Note that $f_{i}(t) \geq 0 \forall t \in \mathbb{R}_{0_{+}}$, for $i \in\{1,2,3\}$, from (19)-(21), and that all of them are monotone decreasing functions which exponentially decrease to zero as time tends to infinity since $p_{2}<p_{1}<0$. Moreover, both $f_{2}(t)$ and $f_{3}(t)$ decrease faster than $f_{1}(t)$ so that the initial constraint $f_{1}(0)-f_{2}(0)-f_{3}(0)=\beta \iota(0) r(0) \geq 0$ implies that $f_{1}(t)-f_{2}(t)-f_{3}(t)=\beta \iota(t) r(t) \geq 0$ $\forall t \in \mathbb{R}_{0+}$. Then $r(t) \geq 0 \forall t \in \mathbb{R}_{0_{+}}$is deduced from the fact that $\iota(t) \geq 0 \forall t \in \mathbb{R}_{0_{+}}$, as it was previously proven.

Finally, the facts that $\iota(t) \geq 0, s(t) \geq 0, r(t) \geq 0$ and $\iota(t)+s(t)+r(t)=1 \forall t \in \mathbb{R}_{0_{+}}$directly imply that $0 \leq \iota(t) \leq 1,0 \leq s(t) \leq 1$ and $0 \leq r(t) \leq 1 \forall t \in \mathbb{R}_{0+}$.

(ii) From (4) it follows that

$$
\begin{aligned}
\frac{\dot{N}(t)}{N(t)}=v-\mu-\rho \gamma \iota(t) & \Rightarrow d[\ln (N(t))]=(v-\mu-\rho \gamma \iota(t)) d t \\
& \Rightarrow N(t)=N(0) e^{(\nu-\mu) t-\rho \gamma \int_{0}^{t} \iota(\tau) d \tau},
\end{aligned}
$$

so the total population $N(t) \geq 0 \forall t \in \mathbb{R}_{0+}$ since $N(0)>0$. Then $0 \leq I(t) \leq N(t), 0 \leq S(t) \leq$ $N(t)$ and $0 \leq R(t) \leq N(t) \forall t \in \mathbb{R}_{0_{+}}$from (5) by taking into account that $0 \leq \iota(t) \leq 1,0 \leq$ $s(t) \leq 1$ and $0 \leq r(t) \leq 1 \forall t \in \mathbb{R}_{0+}$.

(iii) From (5), (23) and (33) it follows that

$$
\begin{aligned}
I(t) & =N(t) \iota(t) \\
& =N(0) \iota(0)\left(\varepsilon e^{p_{1} t}+(1-\varepsilon) e^{p_{2} t}\right) e^{(\nu-\mu) t-\rho \gamma \iota(0)\left(\frac{\varepsilon}{p_{1}}\left(e^{p_{1} t}-1\right)+\frac{1-\varepsilon}{p_{2}}\left(e^{p_{2} t}-1\right)\right)} \quad \forall t \in \mathbb{R}_{0+},
\end{aligned}
$$

and then

$$
\lim _{t \rightarrow \infty}\{I(t)\}=N(0) \iota(0) e^{\rho \gamma \iota(0) \frac{\varepsilon\left(p_{2}-p_{1}\right)+p_{1}}{p_{1} p_{2}}} \lim _{t \rightarrow \infty}\left\{\left(\varepsilon e^{p_{1} t}+(1-\varepsilon) e^{p_{2} t}\right) e^{(\nu-\mu) t}\right\} .
$$

As a consequence, the infected population tends exponentially to zero as time tends to infinity, and then the infection is eradicated from the host population since $p_{2}<p_{1}<0$ and $p_{1}<\mu-v$ from (20). 
(iv) The control law (14) can be equivalently written as

$$
u(t)=\frac{\phi(\overline{\mathbf{x}}(t))+\lambda_{0} \bar{\imath}(t)+\lambda_{1} \bar{s}(t)}{\beta \nu \bar{\imath}(t)}
$$

in the state space defined by $\overline{\mathbf{x}}(t)=[\bar{l}(t) \bar{s}(t)]^{T}$. One can obtain by direct calculations and taking into account (13) that

$$
\begin{aligned}
u(t)= & \frac{1}{\beta \nu \bar{\iota}^{2}(t)}\left\{(\beta-v-\gamma)(v+\omega) \bar{\iota}^{2}(t)-(v+\omega) \bar{s}(t) \bar{\iota}(t)\right. \\
& -[(\beta-\rho \gamma)(v+\gamma+\omega)-\rho \gamma \nu] \bar{\iota}^{3}(t)-(\beta-2 \rho \gamma) \bar{s}(t) \bar{\iota}^{2}(t) \\
& \left.+\bar{s}^{2}(t)+\rho \gamma(\beta-\rho \gamma) \bar{\iota}^{4}(t)+\lambda_{0} \bar{\iota}^{2}(t)+\lambda_{1} \bar{s}(t) \bar{\iota}(t)\right\} .
\end{aligned}
$$

The normalized infected population $\bar{\imath}(t)$ presents a unique maximum value at the time instant $t^{*}$ when $\dot{\bar{l}}\left(t^{*}\right)=\bar{s}\left(t^{*}\right)=0$ as it can be deduced from (26). Furthermore, from (11) and (19), the fact that $\dot{\bar{l}}(0)=\bar{s}(0)=\iota(0)[\beta s(0)+\rho \gamma \iota(0)-v-\gamma]>0$ is implied. As a consequence, one knows that $\bar{s}(t)>0 \forall t \in\left[0, t^{*}\right)$ and $\bar{s}(t) \leq 0 \forall t \in\left[t^{*}, \infty\right)$. In the following, the proof of the non-negativity of $u(t)$ is split into two parts. On the one hand, when $\bar{s}(t)>0$, i.e., $\forall t \in\left[0, t^{*}\right)$, one can deduce that

$$
\begin{aligned}
u(t) \geq & \frac{1}{\beta v \bar{\iota}^{2}(t)}\left\{\lambda_{0} \bar{\iota}^{2}(t)+\lambda_{1} \bar{s}(t) \bar{\imath}(t)+\bar{s}^{2}(t)-[\beta(\beta-v-2 \rho \gamma)+\rho \gamma(v+\gamma)]\right]^{-3}(t) \\
& \left.+(\beta-\rho \gamma)^{2} \bar{\iota}^{4}(t)\right\}
\end{aligned}
$$

by taking into account that $-\bar{s}(t) \geq(\beta-\rho \gamma) \bar{l}^{2}(t)-(\beta-v-\gamma) \bar{l}(t)$ from (29) and the facts that $r(t) \geq 0, \iota(t)=\bar{\imath}(t) \geq 0 \forall t \in \mathbb{R}_{0_{+}}$and $\beta>2 \rho \gamma$ from (19). From (38), if $g_{1}(\beta, v, \gamma, \rho)=$ $\beta(\beta-v-2 \rho \gamma)+\rho \gamma(v+\gamma) \leq 0$, then $u(t)>0 \forall t \in\left[0, t^{*}\right)$. Otherwise, it follows that

$$
\begin{aligned}
u(t)> & \left.\frac{1}{\beta v \vec{\iota}^{2}(t)}\left\{\left[p_{1} p_{2}-g_{1}(\cdot)\right]\right]^{2}(t)+\lambda_{1} \bar{s}(t) \bar{\iota}(t)+\bar{s}^{2}(t)+(\beta-\rho \gamma)^{2} \bar{\iota}^{4}(t)\right\} \\
= & \frac{1}{\beta v \vec{\iota}^{2}(t)}\left\{\frac{\left[p_{1}^{2}-g_{1}(\cdot)\right] \varepsilon-p_{1}[\beta s(0)+\rho \gamma \iota(0)-v-\gamma]+g_{1}(\cdot)}{\varepsilon-1} \bar{\iota}^{2}(t)\right. \\
& \left.+\lambda_{1} \bar{s}(t) \bar{\iota}(t)+\bar{s}^{2}(t)+(\beta-\rho \gamma)^{2} \bar{\iota}^{4}(t)\right\},
\end{aligned}
$$

where $\lambda_{0}=p_{1} p_{2}$ and (20) have been used. From (39), if $p_{1}^{2} \geq g_{1}(\cdot)$, then $u(t)>0 \forall t \in\left[0, t^{*}\right)$ since $p_{1}<0, \varepsilon>1$ and $\beta s(0)+\rho \gamma \iota(0)-v-\gamma>0$ from (19). Otherwise, from (39) it follows that

$$
u(t)>\frac{1}{\beta \nu \vec{\iota}^{2}(t)}\left\{\lambda_{1} \bar{s}(t) \bar{\iota}(t)+\bar{s}^{2}(t)+(\beta-\rho \gamma)^{2} \bar{\iota}^{4}(t)\right\}>0 \quad \forall t \in\left[0, t^{*}\right)
$$

by using the upper-bound $\bar{\varepsilon}_{1}\left(p_{1}\right)$ defined in (22). In summary, $u(t)>0 \forall t \in\left[0, t^{*}\right)$ irrespective of the value of $g_{1}(\cdot)$ and $p_{1}$ whenever $p_{1}$ and $\varepsilon$ satisfy (20) and (21).

On the other hand, when $\bar{s}(t) \leq 0$, i.e., $\forall t \in\left[t^{*}, \infty\right)$, from (37) one obtains that

$$
\begin{aligned}
u(t) \geq & \frac{1}{\beta v \vec{\iota}^{2}(t)}\left\{(\beta-v-\gamma)(v+\omega) \bar{l}^{2}(t)-[(\beta-\rho \gamma)(v+\gamma+\omega)-\rho \gamma v] \bar{l}^{3}(t)\right. \\
& \left.+\bar{s}^{2}(t)+\lambda_{0} \bar{\iota}^{2}(t)+\lambda_{1} \bar{s}(t) \bar{\imath}(t)\right\},
\end{aligned}
$$


where $\beta>2 \rho \gamma$ from (19) has been used. By direct calculations, it follows that

$$
\bar{s}^{2}(t)+\lambda_{0} \bar{\iota}^{2}(t)+\lambda_{1} \bar{s}(t) \bar{l}(t)=\varepsilon(\varepsilon-1)\left(p_{1}-p_{2}\right)^{2} \iota^{2}(0) e^{\left(p_{1}+p_{2}\right) t} \geq 0 \quad \forall t \in\left[t^{*}, \infty\right),
$$

where $\lambda_{0}=p_{1} p_{2}, \lambda_{1}=-\left(p_{1}+p_{2}\right), \varepsilon>1,(26)$ and $\dot{\bar{l}}(t)=\bar{s}(t)$ have been used. Then from (41)

$$
\begin{aligned}
u(t) & \geq \frac{1}{\beta \nu}\{(\beta-v-\gamma)(v+\omega)-[(\beta-\rho \gamma)(v+\gamma+\omega)-\rho \gamma v] \bar{\imath}(t)\} \\
& =\frac{1}{\beta \nu}\left[g_{2}(\cdot)-g_{3}(\cdot) \bar{l}(t)\right] \quad \forall t \in\left[t^{*}, \infty\right),
\end{aligned}
$$

where (22) has been used. If $g_{3}(\cdot) \leq 0$, then $u(t) \geq \frac{(\beta-v-\gamma)(v+\omega)}{\beta v}>0 \forall t \in\left[t^{*}, \infty\right)$ from (43) by using the fact that $\beta>v+\gamma$ from (19). Otherwise, i.e., if $g_{3}(\cdot)>0$, then $h(t)=g_{2}(\cdot)-g_{3}(\cdot) \bar{l}(t)$ reaches its minimum value at the time instant $t^{*}$ when $\bar{l}(t)=\iota(t)$ reaches its maximum value. Such a minimum value is given by

$$
h\left(t^{*}\right)=g_{2}(\cdot)-g_{3}(\cdot) \iota(0)\left[\varepsilon e^{p_{1} t^{*}}+(1-\varepsilon) e^{p_{2} t^{*}}\right]=g_{2}(\cdot)-g_{3}(\cdot) \iota(0) \varepsilon\left(1-\frac{p_{1}}{p_{2}}\right) e^{p_{1} t^{*}},
$$

where the fact that $e^{p_{2} t^{*}}=\frac{\varepsilon p_{1}}{(\varepsilon-1) p_{2}} e^{p_{1} t^{*}}$ since $\dot{\bar{l}}\left(t^{*}\right)=\bar{s}\left(t^{*}\right)=0$ has been used. From introducing the relation between $p_{1}$ and $p_{2}$ of (20) in (44), one obtains

$$
\begin{aligned}
h\left(t^{*}\right) \geq & g_{2}(\cdot)-g_{3}(\cdot) \iota(0) \varepsilon \frac{\beta s(0)+\rho \gamma \iota(0)-v-\gamma-p_{1}}{\beta s(0)+\rho \gamma \iota(0)-v-\gamma-\varepsilon p_{1}} \\
= & \frac{[\beta s(0)+\rho \gamma \iota(0)-v-\gamma] g_{2}(\cdot)}{\beta s(0)+\rho \gamma \iota(0)-v-\gamma-\varepsilon p_{1}} \\
& -\frac{\varepsilon\left(g_{3}(\cdot) \iota(0)[\beta s(0)+\rho \gamma \iota(0)-v-\gamma]+p_{1}\left[g_{2}(\cdot)-g_{3}(\cdot) \iota(0)\right]\right)}{\beta s(0)+\rho \gamma \iota(0)-v-\gamma-\varepsilon p_{1}} .
\end{aligned}
$$

If $p_{1} \leq \frac{g_{3}(\cdot) \iota(0)[\nu+\gamma-\beta s(0)-\rho \gamma \iota(0)]}{g_{2}(\cdot)-g_{3}(\cdot) \iota(0)}$, then $h(t) \geq h\left(t^{*}\right) \geq \frac{\beta s(0)+\rho \gamma \iota(0)-\nu-\gamma}{\beta s(0)+\rho \gamma \iota(0)-\nu-\gamma-\varepsilon p_{1}} g_{2}(\cdot)>0 \forall t \in\left[t^{*}, \infty\right)$ for any $\varepsilon>1$, where the facts that $p_{1}<0, \beta s(0)+\rho \gamma \iota(0)-v-\gamma>0$ and $g_{2}(\cdot)-g_{3}(\cdot) \iota(0)>0$ from (19)-(20) have been used. Otherwise, i.e., if $\frac{g_{3}(\cdot) \ell(0)[v+\gamma-\beta s(0)-\rho \gamma \iota(0)]}{g_{2}(\cdot)-g_{3}(\cdot) l(0)}<p_{1}<0$, then $h(t) \geq h\left(t^{*}\right)>0 \forall t \in\left[t^{*}, \infty\right)$ from using the upper-bound $\bar{\varepsilon}_{2}\left(p_{1}\right)$ defined in (22) for $\varepsilon$. In summary, $u(t)>0 \forall t \in\left[t^{*}, \infty\right)$ irrespective of the value of $g_{3}(\cdot)$ whenever $p_{1}$ and $\varepsilon$ satisfy the constraints in (20) and (21). This fact completes the proof that $u(t)>0 \forall t \in \mathbb{R}_{0_{+}}$irrespective of the values for $g_{1}(\cdot)$ and $g_{3}(\cdot)$ if $p_{1}$ and $\varepsilon$ satisfy the constraints in (20) and (21).

Remark 2 The constraints in (19) relative to the initial conditions and the parameters of the SIRS model are fulfilled for the majority of the epidemic diseases. On the one hand, the disease transmission constant $\beta$ is usually much higher than both the birth rate $v$ and the recovery rate $\gamma$ so that the constraint about $\beta$ in (19) can be considered. On the other hand, at the beginning of the infection propagation, the number of infected individuals is usually small enough and the almost population is susceptible so that the conditions in (19) relative to $\iota(0)$ and $s(0)$ are satisfied.

Remark 3 The process to select the desired poles $p_{1}$ and $p_{2}$, via a suitable choice of $\varepsilon$, is the following. First, once one knows or has estimated the values for the parameters $v, \beta$, 
$\omega, \rho$ and $\gamma$ corresponding to the propagation of a specific epidemics, a value for $p_{1}$ such that $-(v+\gamma)<p_{1}<0$ is chosen. Then the upper-bounds in (21)-(22) for the parameter $\varepsilon$ can be computed in order to choose a value for $\varepsilon$ satisfying $\varepsilon \in\left(1, \varepsilon_{\max }\right)$.

\section{Simulation results}

An example based on the rabbit hemorrhagic disease in the United Kingdom is considered to illustrate the theoretical results presented in the paper. An initial population of $N(0)=$ 1,000 rabbits is used. Such an epidemics can be described by the SIRS model (1)-(4) with the parameter values: $\mu=0.01$ per day (p.d.), $v=0.017$ p.d., $\beta=0.936$ p.d., $\omega=0.0333$ p.d., $\rho=0.9314$ and $\gamma=0.0125$ p.d. Such values are commonly used in the literature $[1,16]$. The main characteristic of such an infection is its high mortality, note the value of the probability of dying from the infection $(\rho=0.9314)$ close to 1 . The initial conditions for the individual populations are given by $S(0)=990, I(0)=10$ and $R(0)=0$.

Two sets of simulation results are presented to compare the time evolution of the populations within the SIRS mathematical model in two different situations, namely: (i) when no vaccination control actions are applied and (ii) if a vaccination based on the described feedback input-output linearization control technique is applied.

\subsection{Epidemics evolution without vaccination}

The time evolution of the system populations in the free-vaccination case, i.e., if $V(t)=0$ $\forall t \in \mathbb{R}_{0_{+}}$is displayed in Figure 1.

The population of rabbits disappears because of the high mortality of the infection as it can be seen in such a figure. As a consequence, a vaccination strategy has to be applied if the eradication of the epidemics is required while guaranteeing the persistence of the rabbits.

\subsection{Epidemics evolution with a feedback control law}

First, note that the considered initial condition and the parameters of the SIRS model for the propagation of the rabbit hemorrhagic disease satisfy the constraints in (19). Then

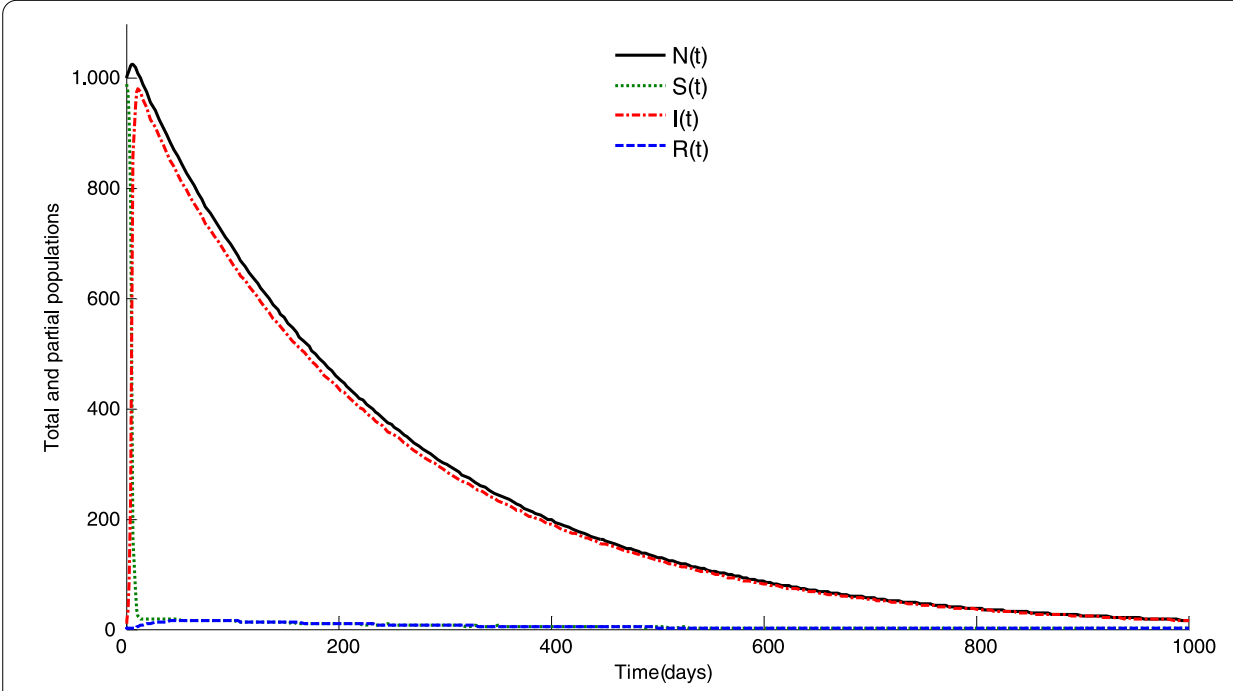

Figure 1 Time evolution of the total and partial (susceptible, infected and recovered) populations without vaccination. 


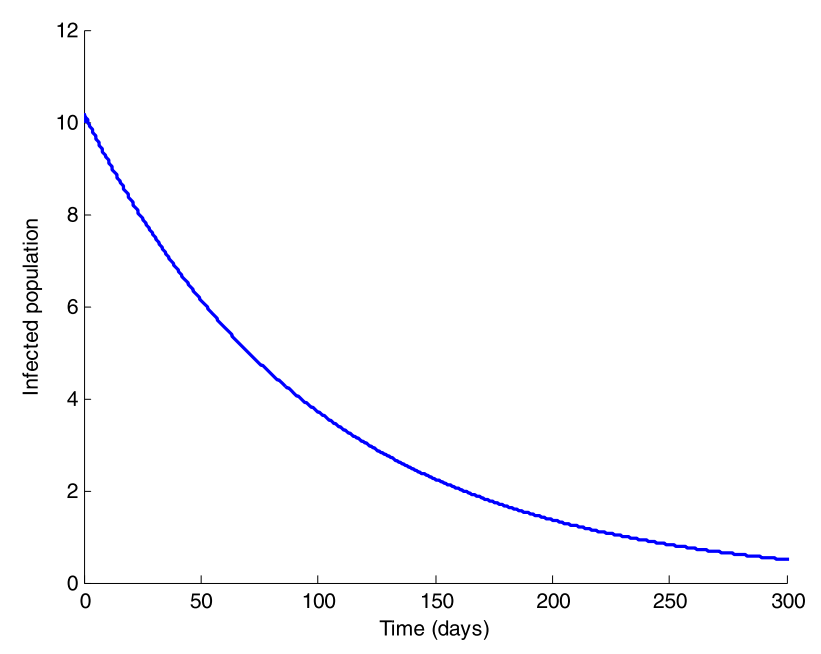

Figure 2 Evolution of the infected population with vaccination.

the control law given by (14), or equivalently written as in (36), can be applied in order to eradicate the epidemics while guaranteeing the non-negativity of the populations and the vaccination control function. The free-design controller parameters $\lambda_{0}=p_{1} p_{2}$ and $\lambda_{1}=-\left(p_{1}+p_{2}\right)$, where $p_{1}$ and $p_{2}$ are the desired roots for the characteristic polynomial $P(s)$ associated with the closed-loop dynamics, are prefixed in the following way. The desired dominant root $p_{1}$ satisfying the constraint $-(v+\gamma)<p_{1}<0$ is chosen, namely, $p_{1}=-v=-0.017$. Then, the upper-bound in (21) for the value of $\varepsilon$ is calculated, namely, $\varepsilon_{\max }=1.0186$. The theoretical results developed in Section 3 prove that a choice of $\varepsilon \in$ $(1,1.0186)$ is sufficient to guarantee the non-negativity of the populations and the vaccination control function in the controlled SIRS model as well as the eradication of the infectious disease. For such a purpose, the value $\varepsilon=1.018$ is chosen. Such a choice for $p_{1}$ and $\varepsilon$ determines the value for the root $p_{2}$ by the relation in (20), namely, $p_{2}=-50.809$. Also, the values $\lambda_{0}=p_{1} p_{2}=0.8638$ and $\lambda_{1}=-\left(p_{1}+p_{2}\right)=50.826$ for the control law are derived from such a procedure. The time evolution of the respective populations is displayed in Figures 2 and 3 while the vaccination control function is shown in Figure 4.

The vaccination control action achieves the control objectives as it is seen in Figures 2, 3 and 4. In this sense, the infection is eradicated from the population since the infected population exponentially converges to zero as Figure 2 shows. Also, all of the partial populations, the whole population and the vaccination control function are non-negative for all time. Such properties are coherent with the results proved in Theorem 3. A consequence of the vaccination control action is that the total population of the rabbits monotonically grows through time in a fast way, like it occurs in the absence of disease, as it can be seen in Figure 3. These simulation results point out the improvement of the use of a vaccination strategy in order to guarantee a suitable growth of the rabbit population against a high-mortality infectious disease.

Remark 4 The conditions in Theorem 3 are sufficient but non necessary to ensure the positivity of the controlled model. Concretely, the upper-bounds $\bar{\varepsilon}_{1}\left(p_{1}\right)$ and $\bar{\varepsilon}_{2}\left(p_{1}\right)$ are sufficient to guarantee the non-negativity of the vaccination function $V(t)$ for all time. However, such upper-bounds can be relaxed in the current example by taking into account the 


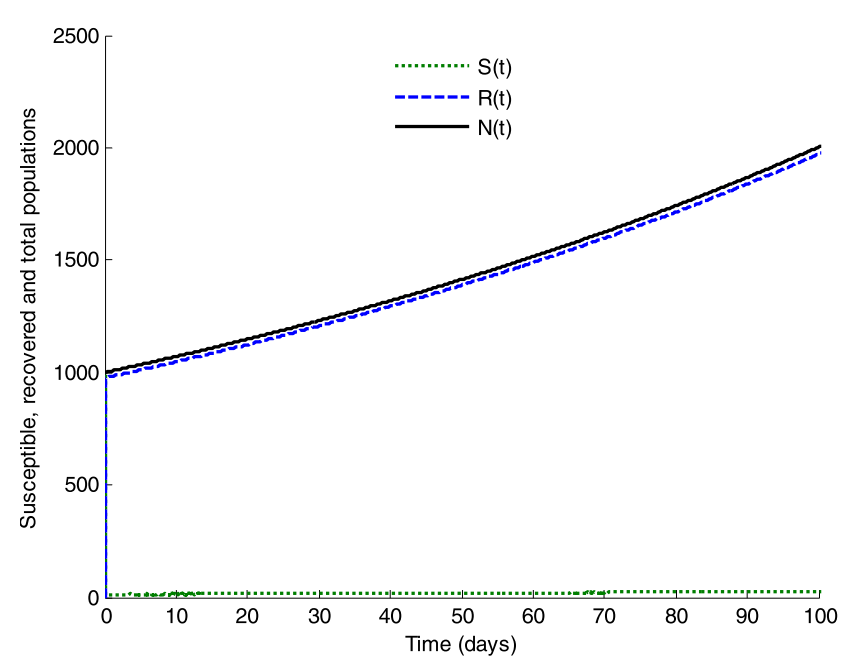

Figure 3 Evolution of the susceptible, recovered and total populations with vaccination.

results obtained from an exhaustive simulation work. In such a work, the non-negativity of the vaccination control function is maintained for all time although the value of the free-design parameter $\varepsilon$ is not smaller than $\varepsilon_{\max }=1.0186$. In this context, the following section analyzes the influence of the parameter $\varepsilon$ in the controlled system dynamics.

\subsection{Influence of the control free-design parameter $\varepsilon$ in the time evolution of the epidemics}

Again, the rabbit hemorrhagic disease is considered for this study and the same dominant pole is chosen for the controlled system dynamics, namely, $p_{1}=-v=-0.017$. Four different values for the parameter $\varepsilon$ are considered, namely, $\varepsilon_{1}=1.018$ (which corresponds to the non-dominant pole of the closed-loop dynamics located in $\left.p_{2}=-50.809\right), \varepsilon_{2}=1.5\left(p_{2}=\right.$ $-1.8455), \varepsilon_{3}=2\left(p_{2}=-0.9313\right)$ and $\varepsilon_{4}=5\left(p_{2}=-0.2456\right)$ for analyzing the influence of such a parameter, and then the influence of the pole $p_{2}$ via the relation in (20), in the controlled system dynamics. Figure 5 displays the time evolution of the infected and total populations for the four different values of $\varepsilon$. The infected population increases until it reaches a maximum value, and then it exponentially decreases to zero as time tends to infinity. Moreover, such a maximum value is smaller and is reached earlier as smaller the parameter $\varepsilon$ is. In this sense, a value for $\varepsilon>1$ and closed to unity is convenient for a fast eradication of the infection from the host population. On the other hand, the influence of $\varepsilon$ in the time evolution of the total population is less appreciable. The total population exponentially increases in a fast way in all cases.

Figure 6 displays the time evolution of the vaccination control function for the different values of $\varepsilon$. One can see that the vaccination control function takes a large value at the initial time instant, and then it decreases until reaching a quasi-stationary regime where its value is maintained below a small threshold for any of the considered values of $\varepsilon$. The magnitude of the vaccination control function at such an initial time instant is larger as smaller the parameter $\varepsilon$ is. Moreover, the vaccination control function shows an oscillatory behavior in the quasi-stationary regime if a value of $\varepsilon$ closed to unity (concretely if $\varepsilon=\varepsilon_{1}$ ) is used while it does not oscillate if $\varepsilon \geq 1.5$. As a consequence, a value 

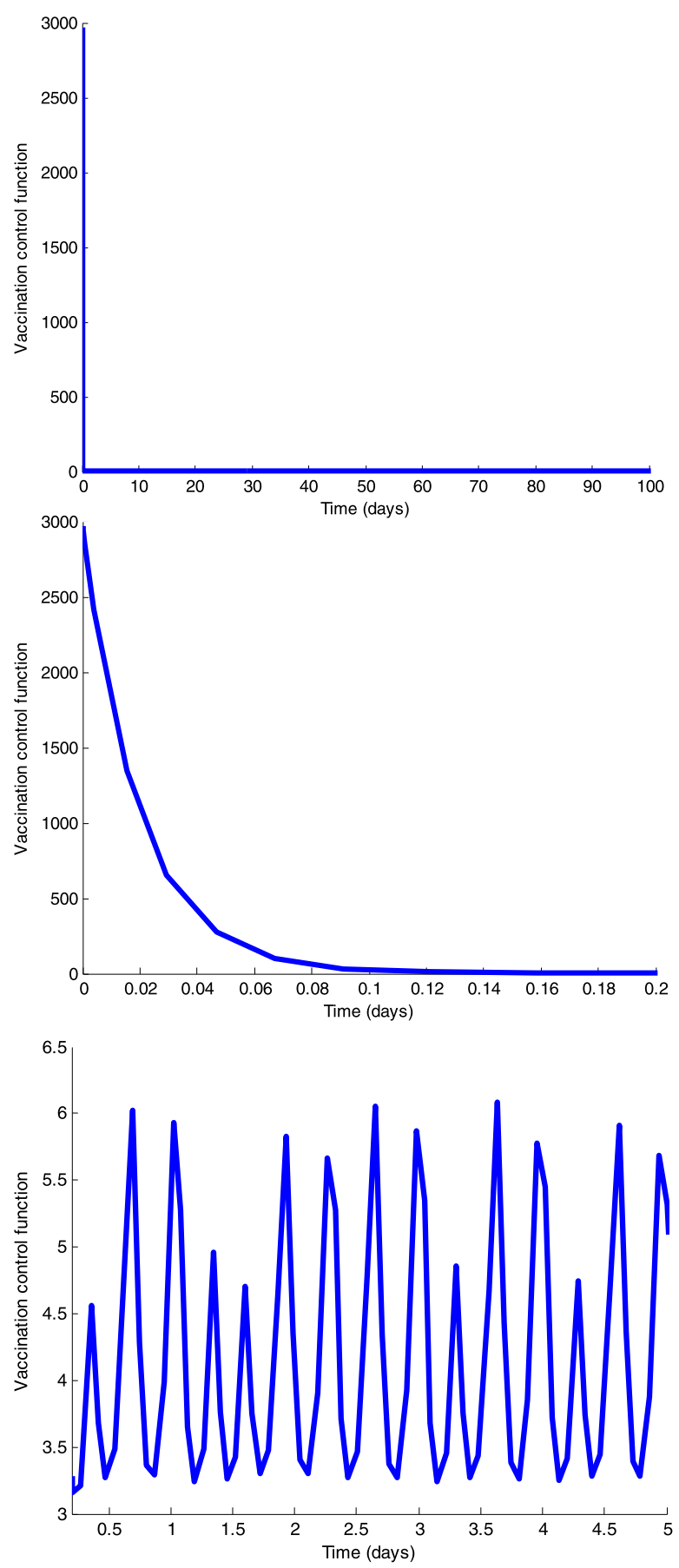

Figure 4 Evolution of the vaccination control function in three different time ranges.

for $\varepsilon$ large enough so that the maximum of the vaccination control function does not exceed a prescribed threshold can be interesting in order to minimize the cost of the treatment of the infection by means of vaccines application. However, a large value of $\varepsilon$ implies a non-appropriate time evolution of the infected population as it has been previously discussed in relation to Figure 5 . As a consequence, a tradeoff between the treatment cost and the evolution of the epidemics has to be taken into account when 

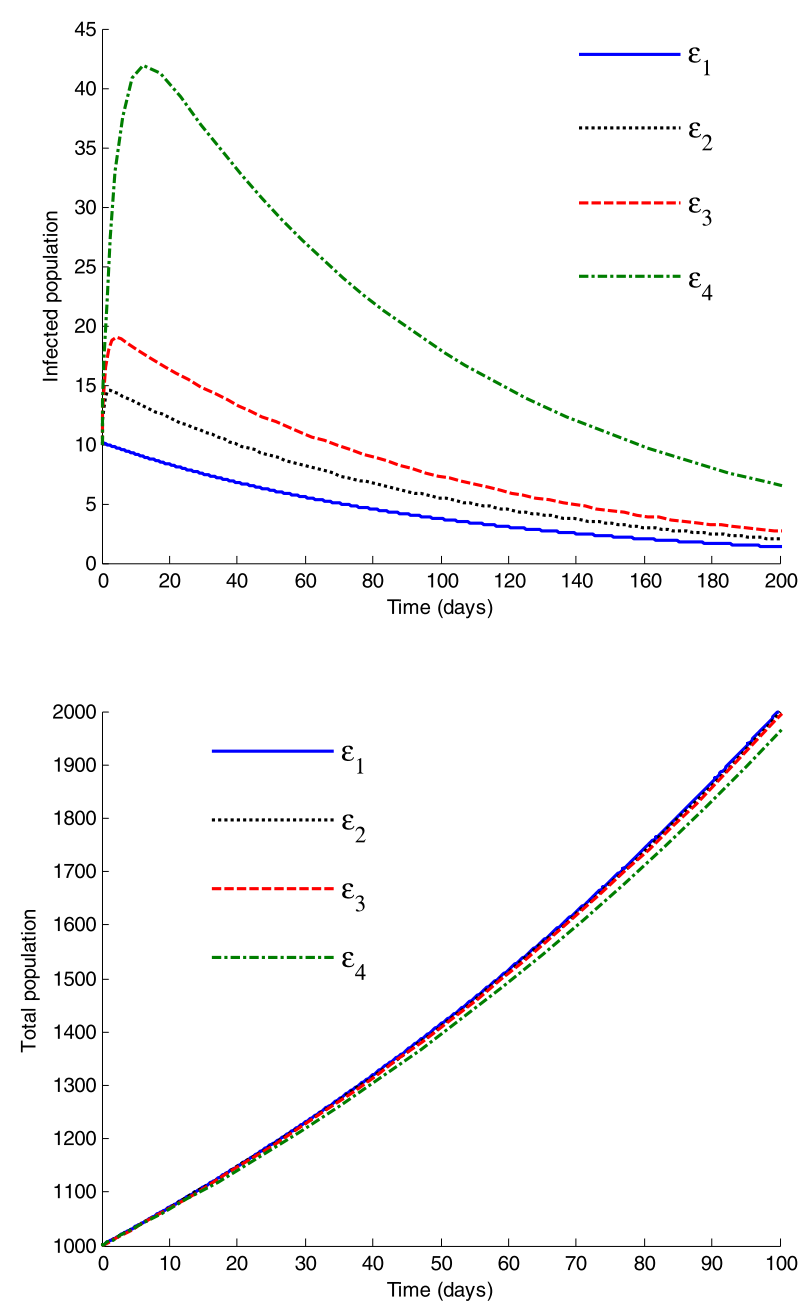

Figure 5 Evolution of the infected and total populations with different values of the parameter $\varepsilon$ used to generate the vaccination function.

choosing the value of the parameter $\varepsilon$ used to generate the vaccination control function.

\section{Concluding remarks}

A vaccination control strategy based on feedback input-output linearization techniques has been proposed to fight against the propagation of epidemic diseases within a host population. A SIRS epidemic model with known parameters is used to describe the propagation of the disease. The total population is time-varying and the model considers the mortality for causes related to the disease. The stability and the positivity properties of the closed-loop system have been proved in the case that true data of the susceptible and infected populations are available. Also, the eradication of the epidemic disease from the host population is guaranteed with such a control strategy. These theoretical results are complemented with some simulation results to illustrate the effectiveness of the proposed approach. Future research into the subject is going to deal with the combination of this control technique with the design of an observer to estimate the susceptible and infected populations when their true data are not available as it usually occurs in a real situation. 

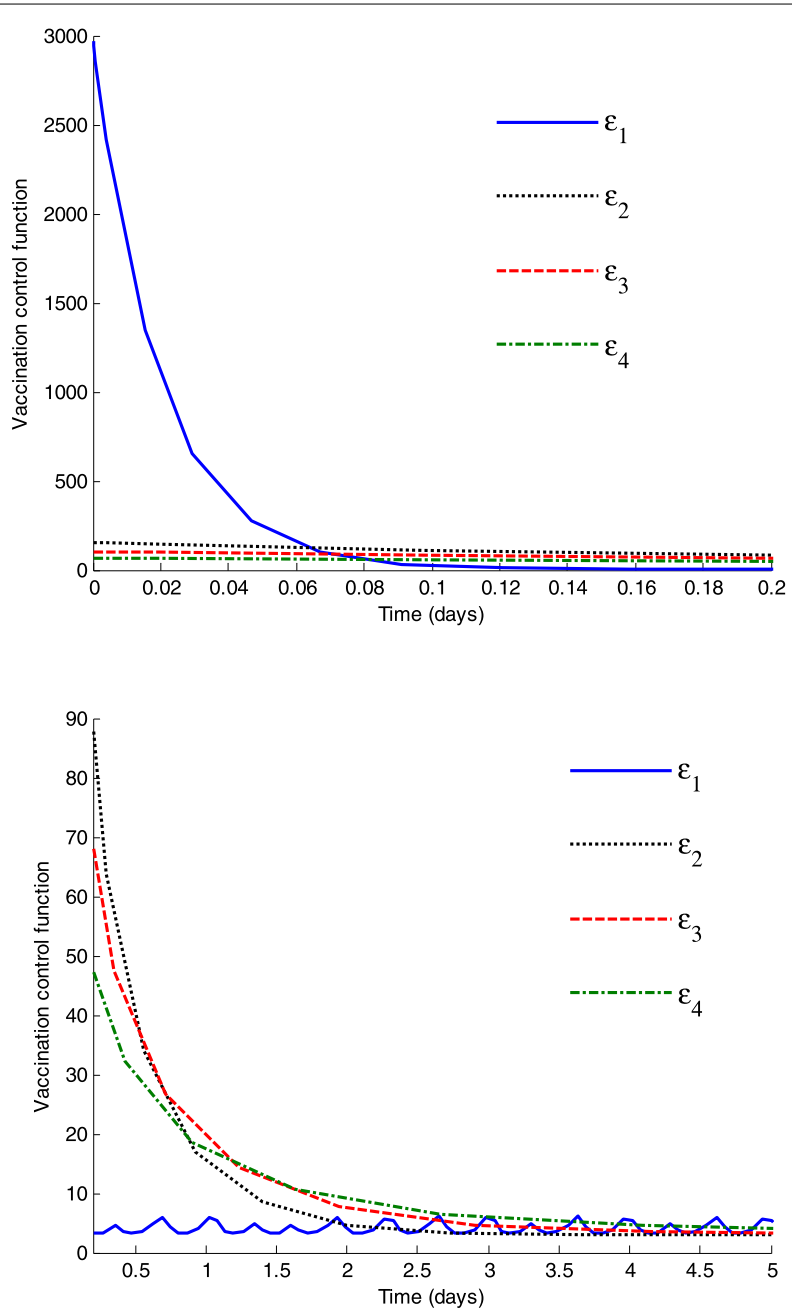

Figure 6 Evolution of the vaccination function with different values of the parameter $\varepsilon$ used to generate the vaccination function.

\section{Competing interests}

The authors declare that they have no competing interests.

\section{Authors' contributions}

All authors contributed equally and significantly in writing this paper. All authors read and approved the final manuscript.

\section{Author details}

'Department of Electricity and Electronics, Faculty of Science and Technology, University of the Basque Country, UPV/EHU, Campus de Leioa, Leioa, Bizkaia 48940, Spain. ${ }^{2}$ Department of Telecommunications and Systems Engineering, Universitat Autònoma de Barcelona, Bellaterra, Barcelona, 08193, Spain.

\section{Acknowledgements}

The authors thank the Spanish Ministry of Economy and Competitiveness for its support of this work through Grants DPI2009-07197 and DPI2012-30651 as well as to the Basque Government for its support through Grants IT378-10, SAIOTEK SPE07UN04 and SAIOTEK SPE09UN12.

Received: 7 February 2013 Accepted: 15 November 2013 Published: 12 Dec 2013

\section{References}

1. Keeling, MJ, Rohani, P: Modeling Infectious Diseases in Humans and Animals. Princeton University Press, Princeton (2008)

2. Li, MY, Graef, JR, Wang, L, Karsai, J: Global dynamics of a SEIR model with varying total population size. Math. Biosci. $160,191-213(1999)$ 
3. Zhang, $Z, W u, J$, Suo, $Y$, Song, $X$ : The domain of attraction for the endemic equilibrium of an SIRS epidemic model. Math. Comput. Simul. 81, 1697-1706 (2011)

4. Greenhalgh, D, Khan, QJA, Lewis, FI: Recurrent epidemic cycles in an infectious disease model with a time delay in loss of vaccine immunity. Nonlinear Anal. 63, 779-788 (2005)

5. De la Sen, M, Agarwal, RP, Ibeas, A, Alonso-Quesada, S: On the existence of equilibrium points, boundedness, oscillating behavior and positivity of a SVEIRS epidemic model under constant and impulsive vaccination. Adv. Differ. Equ. 2011, Article ID 748608 (2011). doi:10.1155/2011/748608

6. Luo, Y, Gao, S, Yan, S: Pulse vaccination strategy in an epidemic model with two susceptible subclasses and time delay. Appl. Math. 2, 57-63 (2011)

7. Zhao, WC, Zhang, TQ, Chang, ZB, Meng, XZ, Liu, YL: Dynamical analysis of SIR epidemic models with distributed delays. J. Appl. Math. 2013, Article ID 154387 (2013). doi:10.1155/2013/154387

8. Alonso-Quesada, S, De la Sen, M, Ibeas, A: A vaccination control law based on feedback linearization techniques for SEIR epidemic models. In: Proceedings of the International Conference on Bioinformatics Models, Methods and Algorithms (BIOINFORMATICS 2012), Vilamoura, Algarve, Portugal, February, pp. 1-4 (2012)

9. Alonso-Quesada, S, De la Sen, M, Agarwal, RP, Ibeas, A: An observer-based vaccination control law for a SEIR epidemic model based on feedback linearization techniques for nonlinear systems. Adv. Differ. Equ. 2012, Article ID 161 (2012). doi:10.1186/1687-1847-2012-161

10. De la Sen, M, Alonso-Quesada, S: On vaccination control tools for a general SEIR-epidemic model. In: Proceedings of the 18th Mediterranean Conference on Control \& Automation (MED'10), Congress Palace Hotel, Marrakech, Morocco, June, pp. 23-25 (2010)

11. Balaguer, P: Similar model reference adaptive control with bounded control effort. Int. J. Adapt. Control Signal Process. 25, 577-592 (2011). doi:10.1002/acs.1222

12. Jugo, J, Lizarraga, I, Arredondo, I: Nonlinear modelling and analysis off active magnetic bearing systems in the harmonic domain: a case study. IET Control Theory Appl. 2, 61 -71 (2008). doi:10.1049/iet-cta:20060461

13. Zamani, I, Shafie, M: Fuzzy impulsive control with application to chaos control. In: Proceedings of the 2009 IEEE International Conference on Fuzzy Systems, Jeju Isl, South Korea, August, pp. 20-24 (2009)

14. Ibeas, A, De la Sen, M, Alonso-Quesada, S: Sliding mode robust control of SEIR epidemic models. In: Proceedings of the 21st Iranian Conference on Electrical Engineering (ICEE 2013), Mashad, Iran, May, pp. 14-16 (2013)

15. Barambones, O, Garrido, AJ: Adaptive sensorless robust control of AC drives based on sliding mode control theory. Int. J. Robust Nonlinear Control 17, 862-879 (2007). doi:10.1002/rnc.1157

16. White, PJ, Trout, RC, Moss, SR, Desai, A, Armesto, M, Forrester, NL, Gould, EA, Hudson, PJ: Epidemiology of rabbit haemorrhagic disease virus in the United Kingdom: evidence for seasonal transmission by both virulent and a virulent modes of infection. Epidemiol. Infect. 132, 555-567 (2004)

17. Jumpen, W, Orankitjaroen, S, Boonkrong, P, Wiwatanapataphee, B: SEIQR-SIS epidemic network model and its stability. Int. J. Math. Comput. Simul. 5, 326-333 (2011)

18. Safi, MA, Gumel, AB: Mathematical analysis of a disease transmission model with quarantine, isolation and an imperfect vaccine. Comput. Math. Appl. 61, 3044-3070 (2011)

19. Zhang, J, Zhen, J: The analysis of epidemic network model with infectious force in latent and infected period. Discrete Dyn. Nat. Soc. 2010, Article ID 604329 (2010). doi:10.1155/2010/604329

20. Isidori, A: Nonlinear Control Systems. Springer, London (1995)

10.1186/1687-1847-2013-364

Cite this article as: Alonso-Quesada et al.: A vaccination strategy based on linearization control techniques for

fighting against epidemic diseases propagation. Advances in Difference Equations 2013, 2013:364

\section{Submit your manuscript to a SpringerOpen ${ }^{\circ}$ journal and benefit from:}

- Convenient online submission

- Rigorous peer review

- Immediate publication on acceptance

- Open access: articles freely available online

- High visibility within the field

- Retaining the copyright to your article 